\title{
Quelles perspectives pour une didactique de la BD en classe de littérature?
}

Nicolas Rouvière et Hélène Raux

\section{(2) OpenEdition \\ 1 Journals}

Édition électronique

URL : http://journals.openedition.org/trema/5209

DOI : 10.4000/trema.5209

ISSN : 2107-0997

Éditeur

Faculté d'Éducation de l'université de Montpellier

Édition imprimée

ISBN : 979-10-96627-07-3

ISSN : 1167-315X

\section{Référence électronique}

Nicolas Rouvière et Hélène Raux, "Quelles perspectives pour une didactique de la BD en classe de littérature? », Tréma [En ligne], 51 | 2019, mis en ligne le 01 avril 2019, consulté le 05 août 2019. URL http://journals.openedition.org/trema/5209; DOI : 10.4000/trema.5209

Ce document a été généré automatiquement le 5 août 2019

Trema 


\title{
Quelles perspectives pour une didactique de la BD en classe de littérature?
}

\author{
Nicolas Rouvière et Hélène Raux
}

1 Maitre de conférences en langue et littérature française à l'université Grenoble-Alpes, Nicolas Rouvière a consacré plusieurs ouvrages à une lecture d'Astérix à la croisée de l'anthropologie et de la psychanalyse $e^{1}$, avant d'initier des travaux visant à développer une didactique de la bande dessinée en classe de littérature. Cet entretien est l'occasion de revenir sur les pistes que ces travaux tracent dans le cadre de la didactique de la littérature.

Hélène Raux: Votre travail de recherche a d'abord porté sur Astérix : vous avez examiné comment les représentations collectives des identités et du politique sont travaillées dans ce que vous désignez comme une littérature de large diffusion. Vous considérez pleinement la bande dessinée comme une forme de littérature?

Nicolas Rouvière: Tout à fait, et je note que cette catégorisation est aujourd'hui assez largement admise par le grand public, le tournant dans les consciences ayant sans doute été l'octroi en 1992 d'un prix littéraire de renommée internationale, le prix Pulitzer, pour le récit graphique Maus d'Art Spiegelman. L'expression "graphic novel » apparue dans le contexte nord-américain et traduite par "roman graphique » en français, traduit assez bien ce changement de perspective et la légitimation littéraire de la bande dessinée - même s'il vise à caractériser un segment commercial, à défaut d'un genre à proprement parler ou de l'ensemble du média lui-même.

Sur le plan scolaire, cette légitimation s'est traduite en France par la publication officielle de listes d'albums recommandés pour les classes, à partir de 1996 pour le collège, et de 2002 pour l'école élémentaire. Je note par ailleurs que les éditeurs scolaires ont pris acte de ce changement dans les représentations culturelles. Depuis 2009, les éditions Magnard proposent dans la collection "Classiques et contemporains » des bandes dessinées en livre de poche, comme pour les classiques de la littérature. Les éditions Gallimard ont emboîté le pas en 2011 avec la collection « Folio BD ».

Sur le plan théorique, le concept pluriel de littératures dessinées a aussi contribué, depuis les années 2000 , à la légitimation littéraire de la bande dessinée. Je reprends 
volontiers ce concept de "littérature dessinée », forgé par l'historien et sémioticien de la BD Harry Morgan, afin de désigner un médium distinct de la littérature illustrée : dans les littératures dessinées, les images constituent un point d'ancrage permanent de la lecture. Un dispositif les distribue stratégiquement sur la page selon une composition porteuse de sens, dispositif qualifié de «spatio-topique » par le sémiologue de la bande dessinée Thierry Groensteen. Par ailleurs, les images des littératures dessinées ont un caractère volontiers narratif, c'est à dire qu'elles présupposent en elles-mêmes un avant et un après, une cause et une conséquence. Enfin ce lien logique et chronologique s'étend à la séquence des images qui s'enchaînent. Lorsque cette séquentialité offre majoritairement trois images par page, on basculerait selon Harry Morgan dans la bande dessinée. Avec ce concept de littératures dessinées, au rebours d'une longue tradition de défiance à l'égard de l'image, le contenu des images et leur dispositif séquentiel sont reconnus dans leur potentialité narrative et leur capacité à piloter un récit, avec ou sans texte.

Hélène Raux : Mais la bande dessinée n'est qu'une des formes de la littérature dessinée...

Nicolas Rouvière : Les littératures dessinées englobent en effet une diversité de formes, allant du dessin humoristique à l'album. Mais ce concept de littérature dessinée me semble opérant pour appréhender la BD aussi bien sur le plan sémiotique, que sur les plans historique et pédagogique. Sur le plan sémiotique, il permet de distinguer le fonctionnement de la BD, dont l'unité séquentielle de base est la vignette, de celui de l'album moderne pour enfant, dont l'unité de base est la double-page. Il rend possible une typologie sémiotique de ces albums, dans leur analogie de fonctionnement avec la bande dessinée. Certains albums pour enfants de Claude Ponti ou d'Yvan Pommaux proposent par exemple des suites de cases légendées (avec ou sans bulle) relevant d'un dispositif BD. A la différence d'autres formes de littératures dessinées, la bande dessinée organise la séquentialité des images majoritairement au sein de la page, autour de triades de vignettes - la vignette en train d'être lue, celle qui précède et celle qui suit. Cette triade constitue en effet une micro-chaîne qui se déplace tout au long de la lecture. Comme l'a montré Thierry Groensteen, c'est un plan de signifiance à part entière, au même titre que la vignette isolée et que le dispositif spatio-topique à l'échelle de la page ou de la double-page. Sur le plan historique, cette approche éclaire les histoires croisées des deux média, jusque dans leurs influences réciproques. Au sein des littératures dessinées, la bande dessinée apparaît ainsi comme la forme médiatique la plus ancienne, son invention remontant aux « histoires en estampes » de Töppfer, publiées dans les années 1830, tandis que l'album pour enfant moderne s'invente progressivement comme objet littéraire et plastique entre la fin des années 1860 et 1919, date de la création par Edy Legrand de l'album Macao et Cosmage. Et sur le plan pédagogique, la définition très large de la bande dessinée proposée par Harry Morgan permet d'ouvrir les représentations des enseignants et des élèves, souvent enferrées dans les stéréotypes de la case, de la bulle ou de la bande, en dépit de l'extrême diversité des productions contemporaines.

Hélène Raux: Enseignant-chercheur chargé de la formation des enseignants, vous vous attachez à une réflexion didactique sur la bande dessinée. Lors du colloque "Lire et produire des bandes dessinées à l'école" que vous avez organisé en 2010, vous parliez du "temps de la méprise" pour caractériser un contexte où la bande dessinée n'est plus méprisée par l'institution scolaire, mais souvent exploitée en vue d'apprentissages qui ne sont pas de son 
ressort, comme quand elle sert d'accroche ludique dans des manuels de grammaire. L'enseignement de la littérature vous semble-t-il concerné par ce type de méprise?

Nicolas Rouvière : S'agissant de l'étude de bandes dessinées dans le cadre du cours de littérature, je parlerais d'écueils, plutôt que de méprise, pour évoquer l'instrumentalisation de la BD au profit d'objectifs de savoirs uniquement textuels ou formels. A partir de ce que j'ai pu observer, dans des classes ou des manuels, j'en pointerais trois.

Le premier est que la lecture de l'image soit minorée voire oubliée, au profit d'un outillage de la lecture textuelle. C'est une réalité assez fréquente dans les séquences de l'école primaire : les élèves sont invités à reformuler les enjeux de l'histoire, à reconstituer un schéma narratif ou un schéma actanciel, mais rares sont les questions qui les amènent à interpréter l'image.

Un autre écueil est d'avoir pour objectif principal de constituer un lexique technique du langage de la BD lui-même, sans lien avec la production du sens. La planche est alors un prétexte à désigner des éléments constitutifs (bulle, case, cartouche, gouttière, onomatopée) et cette acquisition est un objectif en soi, sans que l'effet de sens soit questionné en contexte.

Un troisième écueil, cette fois-ci plus fréquent au collège, est un excès d'observations techniques de la forme multimodale, comme préalable nécessaire à la construction du sens. A titre d'exemple, je renvoie à l'analyse d'un cas extrême que je présente dans BD et enseignement des humanités, un questionnaire que propose le manuel Français $4 \mathrm{e}$ (Magnard 2005), pour le récit complet « Nuages » de Caza - alors même que Magnard est un éditeur en pointe dans ce domaine et que la période 2002-2008 a sans doute été jusqu'ici la plus porteuse pour l'intégration scolaire de la BD : 54 questions ou sousquestions, dont 12 pour la seule première case. Tout y passe : type de plans, profondeur de champ, encadrés narratifs, ellipses, inserts, couleurs, lettrage, taille et forme des cases, raccords visuels, rapports texte-images, etc. Malheureusement tout se passe, avec la bande dessinée, comme si l'un des écueils persistants de l'enseignement de la littérature se trouvait redoublé : un excès d'objets de savoir formels et modélisables est posé comme préalable à la construction du sens, alors que l'expérience vivante et subjective du questionnement devrait être première.

La BD paye sans doute le prix d'avoir émergé dans les programmes scolaires dans les années 1980 et 1990, au plus fort de la dérive techniciste des études littéraires dans le secondaire, quand régnait la lecture méthodique, l'approche par « les types de textes » à l'école primaire, puis par « la maîtrise des discours » au collège, et « la poétique des genres » au lycée. Il est symptomatique du reste que la BD soit classifiée dans les programmes comme un genre, et non comme une forme médiatique.

Qu'il s'agisse de dérives formalistes ou d'une minoration de l'image, dans les ceux cas on est loin d'une lecture littéraire faisant place à l'interprétation et associant participation et distanciation.

Hélène Raux : En quoi consisterait alors une lecture littéraire de la bande dessinée ? Quelle place peut être faite à la dimension visuelle de la BD dans ce cadre?

Nicolas Rouvière : Tout d'abord je voudrais combattre le préjugé tenace selon lequel la bande dessinée serait une lecture « facile » au prétexte qu'elle permettrait de visualiser les personnages, la situation d'énonciation et l'univers référentiel, offrant ainsi tous les indices contextuels pour reconstituer l'intrigue et permettre la compréhension littérale du récit. C'est faire fi du travail d'inférence qu'implique la nature intrinsèquement elliptique des récits séquentiels en image. $\mathrm{La} \mathrm{BD}$ constitue une lecture bien souvent 
«résistante», elle programme sa propre incomplétude, se prête aux effets de polysémie, puise dans le répertoire des représentations collectives pour les mettre au travail et engage une lecture symbolique.

Sa dimension visuelle peut être prise en compte dans l'accompagnement de la réception subjective de la lecture: au rebours d'une approche qui serait d'emblée techniciste, l'étude de récits complets en BD se prête à la création de carnets de lecture en partie "dessinés", dans lesquels les élèves peuvent noter leurs impressions, dessiner ce qui les touche, commenter, coller des images, faire des hypothèses, reconstituer les pensées ou les motivations implicites des personnages, par exemple sous forme de bulles, ou encore porter des jugements, en se dessinant comme personnage-lecteur... La mutualisation des carnets permet ensuite de travailler en classe sur les divergences de ressentis ou de perception du récit, ce qui légitime un retour vers l'œuvre, pour construire le sens ensemble. À la posture de décentration cognitive, permise par le débat, peut succéder une posture d'implication distanciée, à travers l'écriture de nouveaux commentaires dans le carnet, en aval des discussions.

Dans le cadre de la classe, il s'agit, comme pour tout type de texte, de problématiser la lecture, que la problématique soit référentielle, énonciative, psycho-affective, éthique, socio-politique, générique, esthétique ou encore historico-culturelle. La problématisation conduit à développer une lecture-enquête. La particularité de la bande dessinée, c'est que le recueil de traces n'est pas uniquement textuel. Il faut articuler l'ensemble des composantes du langage BD, pour engager le lecteur dans un travail interprétatif de reconstruction du sens.

Dans le livre Bande dessinée et enseignement des humanités, deux articles consacrés à l'étude de l'œuvre intégrale en BD présentent différents dispositifs pour problématiser le récit : comme pour toute lecture, les dispositifs de présentation et de questionnement de l'œuvre sont à définir en fonction des problèmes de compréhension et d'interprétation que cette œuvre pose, mais il semble que la bande dessinée appelle néanmoins d'une façon plus privilégiée certaines stratégies.

La première consiste en la dissociation des composantes textuelle et iconique du récit séquentiel en image: fragmentation de la linéarité du récit, dissimulation d'une vignette, dissociation du texte et de l'image, dissociation entre dialogue et récitatif, fragmentation des planches sous forme de puzzle, etc. Le but est à chaque fois d'abstraire un élément porteur de sens, afin que l'effet de sa réapparition soit plus saillant.

A l'inverse, l'étude du médium appelle de façon tout aussi privilégiée un travail de mise en lien et d'analogie thématique ou structurelle : à l'échelle d'une section de pages, à l'échelle de la planche, ou bien à l'échelle plus réduite des bandes ou des vignettes. Le but de ces analogies est alors de convoquer simultanément toutes les composantes textuelles, iconiques et plastiques du langage BD, pour montrer comment leur articulation participe à l'effet de sens. Ce travail de mise en lien peut conduire à la création de découpages et de mises en page inédits. Il ne se limite pas non plus à l'album, mais trouve un prolongement dans la mise en réseau avec d'autres supports littéraires ou iconiques (peintures, photographies, romans, BD, etc.). Toutes ces activités n'ont bien évidemment de sens que si elles visent à mettre les élèves en situation de résolution de problèmes de compréhension ou d'interprétation et si elles sont corrélées à de réels objectifs d'apprentissage. 
Hélène Raux: Vous avez testé de tels scénarios dans des classes, quel bilan en avez-vous tiré ?

Nicolas Rouvière : Je renvoie à un article publié dans Les Cahiers pédagogiques $\mathrm{n}^{\circ} 506$ pour montrer comment la mise en évidence d'analogies de composition, entre deux planches du tome 2 de la série Angelot du lac a pu déboucher en classe de CM2 sur la construction d'un sens implicite. Il s'agit de deux scènes de première rencontre, l'une d'Angelot, le jeune héros, avec Agnès, une jeune recluse destinée à un riche mariage, l'autre d'Angelot avec un léopard en cage, destiné à être offert au futur époux. Les élèves ont repéré les analogies entre les multicadres, les enchaînements des vignettes et leur composition. Par-delà les mises en scène successives, les élèves ont cerné la force de l'empathie du garçon pour la bête, dont il partage l'aspiration à la liberté, alors qu'il est progressivement déçu par Agnès, qui préfère à l'aventure son sort contraint de future épouse. Une double planche muette représente la mort de l'animal sous les flèches des soldats, en parallèle avec la réaction convulsive du héros. À la question "Que représente le léopard pour Angelot ?", les élèves ont répondu «Ils sont comme des frères ", " ils sont jumeaux », " c'est son ami », « le léopard lui a sauvé la vie ». Le fait qu'il symbolise la liberté à laquelle Agnès a finalement renoncé, en revanche, n'a pas émergé dans les réponses des élèves, qui sont restés attachés au stéréotype de la rencontre amoureuse entre les deux enfants. Compte tenu de la complexité de la relation entre les trois personnages, il est bon que le sens reste ouvert, et que les différentes interprétations puissent s'énoncer et coexister dans la classe, comme autant d'éléments témoins de la richesse de l'œuvre étudiée.

Au final, la comparaison entre les deux scènes de rencontre, ainsi que l'étude du face à face entre Angelot et la bête, puis celle de la scène muette finale, ont permis de changer le regard des élèves sur la narration en bande dessinée. Suite à cette séquence, plusieurs ont confié qu'ils étaient plus attentifs à la taille des cases, à la façon dont les personnages étaient présentés, plus ou moins près, et aux ressemblances dans la disposition des vignettes, sur une même planche, ou d'une planche à l'autre. Il s'agit là d'un gain considérable pour la lecture. Les élèves quittent le simple niveau de la fiction pour passer à l'examen distancié de la narration, avec ses procédés et ses stratégies. La BD facilite cette prise de distance, car les procédés narratifs sont littéralement visibles, pour peu que l'on exerce le regard. Les élèves ont surtout découvert que le pilotage du récit par l'image était lourd d'effets implicites, ouvrant la voie à une lecture symbolique.

Hélène Raux: La lecture de bandes dessinées requiert-elle une formation du lecteur spécifique pour exercer ce regard?

Nicolas Rouvière : Une acculturation générale au média me semble indispensable, pour ouvrir les représentations des élèves : à la diversité des univers de fiction, des genres, des styles graphiques, des thèmes, au processus de création, à l'histoire du médium, à ses différentes sphères géographiques et culturelles dans le monde, mais aussi, pourquoi pas, à la réflexion sur les frontières sémiotiques de la $\mathrm{BD}$ avec d'autres formes d'expression. Cela peut passer, de l'école primaire au lycée, par différentes activités, en profitant ou non des modules interdisciplinaires, dans deux directions : d'une part pour identifier et manipuler le langage de la BD, d'autre part pour connaître l'histoire de la bande dessinée et sa diversité.

Pour faire découvrir la diversité de la bande dessinée, les activités éprouvées pour familiariser les élèves avec le monde des livres en général peuvent être déclinées sur la bande dessinée : à partir du CE1, un dispositif comme celui de la « ronde des livres » par 
exemple, dans lequel l'enseignant présente une caisse d'albums parmi lesquels les élèves lisent une sélection de leur choix puis indiquent un jugement de goût sous forme de pictogramme dans un tableau collectif, et présentent le livre qu'ils ont lu, pour que s'amorce une discussion; des visites d'expositions aident à faire connaitre l'histoire de la bande dessinée et sa diversité dans le monde, des rencontres avec des auteurs amènent à envisager la genèse d'un album...La diversité des genres et des styles peut être mise en évidence par un tri de planches ou d'albums: des groupes d'élèves reçoivent un corpus de planches ou d'albums mêlant des univers de fiction, des thèmes, des genres, des formats et des styles différents et doivent essayer de classer les supports, puis les choix sont mis en commun et justifiés, des affiches sont élaborées selon ces 5 catégories, qui seront complétées d'exemples supplémentaires au fil de l'année. Ce ne sont que quelques propositions visant à ouvrir les représentations des élèves mais cette dimension me semble fondamentale.

Pour travailler plus spécifiquement le langage de la BD et le manipuler, on peut créer des situations problèmes sur les frontières de la BD avec d'autres formes expressives comme les caricatures séquentielles, le roman-photo, la peintures d'images itératives, les albums pour enfants, les fresques séquentielles, etc. Un tri de documents icono-textuels mêlant image et texte avec compartimentage amènera par exemple à dégager des critères de reconnaissance d'une bande dessinée, dans laquelle le récit est en particulier piloté par des images qui se suivent.

Dans une perspective de formation, l'exploration du langage de la BD est un axe important, pour la connaissance et la maîtrise des codes sémiotiques. Cependant je mettrais de nouveau en garde contre une pédagogie fondée sur une liste de procédés formels, qui seraient à étiqueter et enseigner les uns à la suite des autres, de façon décontextualisée.

La planche (en commençant par les planches muettes) représente une unité à bonne échelle pour l'analyse. Assez vite, avec quelques notions (cadres, site, plan, angle de vue), les élèves aiguisent leur regard, de telle sorte que l'on peut construire des analyses de planche très intéressantes. Lorsque l'on a pour objectif d'étudier un procédé formel précis, je recommanderais de le faire dans des contextes diversifiés, afin d'éviter un figement des représentations quant aux effets associés. La variété des emplois et des effets d'un même procédé peut être notée au fil des rencontres sur une affiche dédiée.

Il me semble essentiel également de privilégier la manipulation. L'activité de puzzle, où chaque pièce correspond à une vignette, est riche à cet égard pour s'approprier les codes sémiotiques. Les indices à disposition des élèves sont de plusieurs ordres: linguistique, à travers le cours logique des récitatifs et des dialogues; figuratifs, à travers la représentation enchaînée des actions ; et compositionnelles, à travers le format et la taille des cases, qui viennent pré-cadrer les possibilités de mise en page. C'est la justification des élèves qui importe avant tout, qui peut enrichir les stratégies de lecture de BD en les explicitant, en pointant l'importance des différents systèmes de signes. C'est dans ces phases d'échange, où les conditions de l'appropriation sont optimales, que peuvent être introduits pour la première fois les termes techniques du langage $\mathrm{BD}$ et posés les premiers éléments de structuration, en vue d'une institutionnalisation ultérieure des savoirs.

D'autres activités s'avèrent porteuses. Par exemple pour le travail des ellipses, les activités d'augmentation ou de réduction de planches, ou de montages de planches inédites à partir de vignettes existantes ; ou encore, pour réfléchir à la mise en page, 
l'appariement d'un extrait de synopsis avec un multicadre au choix, à remplir, pour comparer ensuite les choix des élèves avec celui de l'auteur. Cela peut se faire à l'échelle de la case, pour trouver par exemple le motif iconique et le type de plan qui peuvent correspondre à une bulle donnée, dans une case vide. Pour appréhender la séquence, une possibilité est de transformer une image narrative en bande dessinée : dans une classe de CM2, à partir du tableau Le tricheur au carré d'as de Georges de La Tour, les élèves ont répondu à la question « à votre avis, qui triche, et qui est avec qui ?» en créant une courte bande dessinée à partir de fragments du tableau reproduits, grossis ou réduits, qu'ils ont sélectionnés, associés, enrichis graphiquement et textuellement. Sans le savoir, ils ont ainsi mis en œuvre des opérations essentielles $\mathrm{du}$ processus de création d'une BD: mettre en relation des dessins pour créer une histoire et articuler les vignettes dans une mise en page significative.

De manière générale, l'appropriation des procédés expressifs passe par la production.

Hélène Raux : Le lien entre lecture et production est précisément au cœur des démarches de la lecture littéraire. Or ce point semble poser problème avec les bandes dessinées : les enseignants se disent embarrassés par le dessin, qu'ils peinent à accompagner dans le cadre du français et qu'ils mènent parfois plutôt en arts visuels à l'école, ou en interdisciplinarité avec les arts plastiques au collège. A quelle production peut donner lieu une séquence de littérature appuyée sur une bande dessinée?

Nicolas Rouvière : Il est possible d'articuler lecture de BD et production de BD comme pour toute étude d'un texte littéraire. C'est du reste une voie majeure, à la fois pour construire la compréhension du récit, développer une posture interprétative et s'approprier le fonctionnement du média. Et rien n'interdit d'avoir une ambition un peu plus haute que celle de faire compléter des bulles vides ou des planches à trous, en visant la production d'une ou plusieurs planches.

Pour exemple, je renvoie ici à la création, par des élèves de CM1, d'épisodes intermédiaires visant à s'insérer dans les albums Petit vampire va à l'école et Le tour de Gaule d'Astérix. Le premier a conduit à problématiser la tonalité du récit et le second à s'approprier le schéma actantiel ainsi que des éléments de la structure narrative.

Dans le cadre du cours de français, les compétences à travailler pour la production de planches relèvent davantage du découpage du récit que de la mise en page, c'est à dire de «l'arthrologie », plutôt que de la «spatiotopie » selon les catégories de Thierry Groensteen.

Si l'écriture d'un synopsis mobilise des compétences d'écriture scolaires traditionnelles, l'écriture d'un scénario, en revanche, suppose que les élèves aient la capacité spécifique de pré-visualiser ce qu'ils racontent et mettent en scène : le site, la taille et la forme des cases, les types de plans, le contenu figuratif, etc. Or cette compétence particulière, qui consiste véritablement à "penser en images " ne fait l'objet bien souvent d'aucun apprentissage préalable. Ainsi des élèves performants dans l'écriture du synopsis peuvent-ils se trouver démunis lorsqu'il s'agit de passer à l'étape suivante.

La production de planches articulée avec l'étude du Tour de Gaule d'Astérix en classe de CM1, nous a ainsi conduit à inventer le dispositif de « l'écriture post-it » sur de grandes feuilles A3, afin de fusionner les étapes du synopsis et du scénario. Sur chaque post-it d'une même couleur, les élèves doivent écrire une action et une seule, et inventer ainsi leur histoire, en faisant progresser l'action de post-it en post-it. Ce principe aide les élèves à pré-visualiser leur récit, selon l'équivalence tacite : 1 post-it $=1$ case. Par ailleurs les post-it sont déplaçables, supprimables et désinhibent les élèves par le jeu de manipulation ludique et concret qu'ils suscitent. Cela permet de changer le rapport à 
l'écrit et de travailler à plusieurs, en discutant oralement de la nature et de l'orientation du récit. Dans un second temps, les élèves superposent aux actions consignées, un post-it d'une autre couleur, pour inscrire le texte (récitatif, onomatopée, dialogues); enfin, dans une troisième étape, un troisième post-it d'une couleur encore différente, est superposé pour la description plus précise de l'image, en vue du dessin à venir (plan, décors, cadrage, etc.). Grâce à ce dispositif, le synopsis s'invente ou se réinvente en même temps que le scénario s'écrit, case après case. Non seulement on évite la lourdeur de leur enchaînement, mais on observe que se développent le plaisir et l'émulation. Les élèves anticipent même le site, la taille et la forme des cases en collant des post-it entre eux, pour former des cases à leur convenance. Planification, mise en texte et révision ne sont plus des étapes chronologiques, mais se fondent dans un seul et même processus dynamique.

Hélène Raux : Le dessin devient donc accessoire dans ce dispositif ?

Nicolas Rouvière : Dans le cadre du cours de français, je dirais que la question du dessin est secondaire. Si elle peut enthousiasmer certains élèves, d'autres en revanche montrent une grande inhibition. Le dispositif de l'écriture post-it relève en fait d'une conception scénaristique de la production en BD. De ce point de vue, l'intégration scolaire du 9e art est une chance pour la discipline français. Il serait temps en effet que l'apprentissage du scénario et de la mise en intrigue devienne un objet d'étude à part entière au sein de la discipline. Cette approche différente de la production d'écrit permet de révéler des compétences narratives que l'on ne soupçonne pas toujours. Ainsi des élèves plus en difficulté dans des productions écrites traditionnelles peuvent se découvrir un véritable talent de scénariste, par leur capacité à pré-visualiser et mettre en scène avec ces petits papiers ce qu'ils veulent raconter - cela ne signifie pas que c'est une activité facile, et d'autres élèves rencontrent au contraire de grandes difficultés avec ce travail ; mais en diversifiant les modalités d'écriture, on multiplie les chances de construire un rapport à l'écrit positif pour tous. Les objectifs du dispositif "post-it» restent pleinement intégrés au cadre du cours de français: les grandes feuilles ainsi obtenues sont des supports tout à fait évaluables en termes de cohérence et de pertinence narratives.

Une fois cet objectif posé, le dispositif peut s'articuler de manière différenciée à la réalisation graphique. Pour les élèves qui ont besoin tout d'abord de «crobarder » pour inventer leur histoire, l'écriture post-it clarifie le plus souvent le récit à leurs propres yeux et les incite à reprendre plus tard sous une forme différente leur version dessinée. A contrario, il est très difficile d'obtenir des élèves qu'ils redessinent leur planche, si celle-ci est déjà entièrement «crobardée ». La phase d'écriture post-it, qu'elle intervienne d'emblée dans le dispositif, ou après un premier découpage dessiné, s'avère toujours pertinente.

Concernant le dessin lui-même, à l'école élémentaire, on gagne à considérer le dessin en $\mathrm{BD}$ comme un dessin d'idée, plus que comme un dessin figuratif à visée mimétique. A partir de là, on peut apprendre aux élèves à dessiner les personnages à partir de formes géométriques simples, qu'il s'agisse de la silhouette générale du corps, ou des éléments d'expression du visage. J'ai eu l'occasion d'exposer un dispositif de ce type au cycle 3 à propos de l'étude d'Astérix et de Petit vampire, basé sur un principe très efficace de répétition et d'ajout en 8 étapes, réalisé en direct au tableau par le professeur et reproduit en même temps par les élèves. Le résultat obtenu est souvent pour eux une révélation. Dans le second degré, ce travail peut être en partie externalisé, comme un 
travail personnel à la maison, ou dans un cadre interdisciplinaire avec les arts plastiques.

Un autre point concerne l'intégration des bulles et des cartouches dans la planche. Le plus simple consiste à superposer une feuille de papier calque, afin de pouvoir effectuer un premier jet où les contours des bulles et cartouches seront tracés et complétés du texte correspondant. Ce support permet une correction par le maitre à même la feuille de calque, autour de critères de spatialité, lisibilité, cohérence, syntaxe et orthographe, avant insertion sur la planche.

Un autre moyen de contournement des difficultés graphiques consiste à utiliser l'outil numérique, comme le logiciel Comic life, le module «Bidule » proposé par les éditions Milan dans les CD interactifs Mobliclic, pour les enfants de 7 à 13 ans, ou la banque d'outils en ligne Pixton. Différents menus déroulants déclinent des personnages dans toutes les attitudes, des éléments de décor, des bulles et cartouches de toutes sortes. Il faut choisir un multicadre, cliquer sur une case, y faire glisser ces éléments, puis écrire à l'intérieur. Le plus difficile est le cadrage pédagogique de l'activité : l'attirance des élèves pour la banque des images disponibles à insérer dans les cases les conduit très souvent à composer une suite de cases, plutôt qu'une histoire cohérente. C'est pourquoi il est préférable de mener prioritairement un travail de planification, où l'écriture postit peut retrouver une fois encore tout son rôle.

Hélène Raux: Que pensez-vous de l'importante production éditoriale d'adaptations d'œuvres littéraires? Quel intérêt voyez-vous à ces adaptations pour l'enseignement de la littérature?

Nicolas Rouvière: L'engouement des éditeurs de BD pour les adaptations s'explique parfois par l'ambition de trouver un débouché auprès des prescripteurs que sont les acteurs du monde éducatif (parents, animateurs, bibliothécaires, professeurs ou inspecteurs), avec l'idée que le médium serait une voie facilitante pour construire une culture patrimoniale commune. Nous avons déjà évoqué l'illusion selon laquelle la BD serait une lecture intrinsèquement "plus facile »; il convient d'ajouter ici le risque de renforcer les hiérarchies culturelles, si l'œuvre adaptée est pensée uniquement comme un marchepied vers l'œuvre originale. Un préjudice corollaire serait de développer en classe une narratologie comparative, qui n'aurait pour but que de mesurer le degré de fidélité à l'œuvre source.

Refaire le parcours qui conduit de l'œuvre d'origine à l'œuvre d'arrivée peut fausser le regard sur cette dernière. La fidélité, du reste, ne semble pas constituer un critère d'évaluation pertinent en soi, pour juger de la qualité d'une adaptation. Autant étudier l'œuvre adaptée pour elle-même, comme si c'était une œuvre originale. Cela permet de tracer une ligne de partage entre les adaptations résultant d'un vrai projet d'auteur et celles qui n'ambitionnent pas de propos spécifique. C'est alors que la comparaison peut trouver dans un second temps toute sa pertinence. La question est moins de savoir comment l'œuvre littéraire est adaptée en BD, que de comprendre selon quelles modalités esthétiques et avec quelles visées une œuvre en bande dessinée peut raconter une histoire peu ou prou similaire. Du reste, lorsque les systèmes de valeur diffèrent quelque peu entre l'œuvre source et l'œuvre seconde, cela active les mécanismes de compréhension et d'interprétation. 
Hélène Raux: L'adaptation de textes en BD par les élèves favorise-t-elle aussi ce travail d'interprétation?

Nicolas Rouvière: Plusieurs expérimentations tendent à montrer que cette pratique donne une réelle impulsion à l'exploration du texte source pour construire la compréhension. Elle oblige les élèves à plonger dans le texte, à le découper, distinguer les différentes factures de discours, les instances énonciatives, séquencer les actions, les dialogues ou les passages descriptifs. Le processus d'adaptation engage par ailleurs chez les élèves un effort d'élucidation lexicale et de représentation mentale pour se forger des images. Effort qui est souvent étayé par des recherches documentaires pour enrichir la lecture et nourrir leur projet.

Cependant, en ce qui concerne la lecture proprement littéraire, le bénéfice pour l'interprétation du texte source semble limité. En effet, une fois que les élèves ont dégagé une note d'intention, il apparaît que leur retour au texte est le plus souvent instrumental pour servir leur projet particulier. Le texte devient alors un réservoir, utile à d'autres fins qu'à sa propre lecture, dans le sens d'une réduction et d'une simplification. L'adaptateur écarte désormais tout ce qui ne sert pas son propre projet, que les éléments soient figuratifs ou pas. Ce mouvement semble inhérent à l'adaptation, indépendamment d'une volonté ou pas de fidélité. Lors de la présentation ultérieure des planches, le travail comparatif avec le texte source peut s'avérer également assez pauvre. Il n'enrichit pas véritablement la lecture. Les élèves voient le texte original à travers le prisme de leur propre adaptation, pour étayer leur projet, quel qu'il soit. Le gain le plus notable est certes la mise en mouvement de l'activité fictionnalisante du lecteur : en particulier la "cohérence mimétique ", lorsque le lecteur établit des liens de causalité entre les actions, ainsi que le « jugement axiologique » sur les personnages, qui peut déborder le cadre de la fiction pour donner lieu à une prise de position sur le monde ; enfin l'activité fantasmatique, lorsque le lecteur (re)scénarise des éléments d'intrigue à partir de son propre imaginaire. Mais cette mise en mouvement intervient en amont du processus de l'adaptation lui-même, après la lecture découverte, lorsque l'on suscite par exemple un jeu d'images associatives. La transmodalisation apporte en aval peu de gain supplémentaire sur ce plan.

Finalement c'est moins la lecture littéraire du texte source, qui se trouve enrichie, que le recul réflexif des élèves sur leur propre activité de production du sens. En effet l'analyse qu'ils font de leur propre planche montre souvent une articulation explicite entre une intention signifiante, des procédés de composition et des effets de sens (même s'il s'agit d'un discours reconstruit). De fait, il est plus facile aux élèves d'adopter cette posture avec leurs propres productions, car ils ont un sentiment de maîtrise et de pleine légitimité à parler de ce qu'ils ont voulu faire. La BD semble un médium particulièrement propice à l'adoption de cette posture. Car pour peu que l'on exerce le regard des élèves, les procédés que sont le choix d'un multicadre, la taille, la forme et le site des vignettes, ou encore les jeux d'échelle de plan ou les angles de vue, sont littéralement visibles, dans la planche. C'est parfois pour les élèves une révélation. Ils peuvent constater par eux-mêmes qu'ils apprennent à voir. En l'occurrence les élèves décollent de la fiction pour voir comment est agencé ce qui est montré et raconté. Ils peuvent très rapidement raisonner en termes de stratégie narrative, ce qui constitue un gain important.

Hélène Raux: A propos des adaptations, vous suggériez que dans l'abondante production, toutes les propositions ne se valent pas. Quels conseils donneriez-vous aux enseignants en 
matière de corpus, de choix d'une BD ? Beaucoup hésitent par exemple à proposer des mangas à leurs élèves...

Nicolas Rouvière: Comme dans le choix de tout corpus littéraire à l'école, il est important d'être touché par une œuvre, sa forme et son propos. Mais la scolarisation d'une œuvre pose la question de sa portée éducative et des objectifs d'apprentissage visés, adaptés au niveau de classe concerné. C'est la pierre de touche de tout choix, en veillant encore une fois à ce que l'étude ne soit pas instrumentalisée au profit des seuls objectifs formalistes d'outillage de la lecture. A partir de là, il n'y a pas de raison de limiter le choix à un genre, un style ou une sphère culturelle, même si notre proximité est a priori plus grande avec la tradition franco-belge. La difficulté pour les enseignants est surtout de se fournir en exemplaires, ce qui dans les faits, limite considérablement le choix. On fait généralement avec ce qui est disponible à moindre coût, en tablant parfois que certains exemplaires seront déjà possédés à la maison. Engager les élèves à acheter une $\mathrm{BD}$ au format poche peut exposer à la critique de certains parents, à la fois pour le prix de l'objet et sa nature-même. Lorsque l'on doit combler le manque par des photocopies ou recourir à la vidéo-projection, au risque d'enfreindre une limite légale, on touche le premier obstacle de la scolarisation de la BD, qui est d'abord matériel.

Sinon, pour s'aider dans leur choix, je recommande aux enseignants de consulter des organes de médiation : parmi les ressources spécialisées en $\mathrm{BD}$, avec le site de la Cité Internationale de la Bande Dessinée et de l'Image, les revues en ligne Neuvième Art, Comicalités, du9 l'autre bande dessinée, les magazines DBD et Bo Doï, les sites Bdzoom et Cases d'histoire ou encore le magazine papier Casemate, on peut consulter la base nationale en ligne L@bd du CNDP, qui propose des notices pour plusieurs milliers d'albums avec des pistes pédagogiques, des indications de niveaux, ainsi que des dossiers thématiques. La Revue des livres pour enfants propose aussi des sélections de bandes dessinées. Et plus largement la BD occupe une place croissante dans les médias consacrés aux livres, le Magazine littéraire propose des critiques de BD, des auteurs de BD sont invités dans des émissions littéraires à la radio ou à la télévision...

Globalement, les ressources pour s'orienter dans la production se sont multipliées, ce qui accompagne aussi la légitimation du médium dans la sphère culturelle. Même si au niveau des programmes les choses ne sont pas linéaires, avec des avancées et des reculs en matière de reconnaissance de la $\mathrm{BD}$, un mouvement culturel de fond est en marche. Les choses semblent progressivement se mettre en place, pour qu'un jour, une œuvre en bande dessinée soit inscrite au programme de lettres du baccalauréat. A mon avis, ce n'est qu'une question de temps. Ce sera alors une étape majeure pour l'histoire du médium, mais aussi pour l'histoire de la discipline elle-même.

\section{BIBLIOGRAPHIE}

Morgan, H. (2003). Principes des littératures dessinées, Angoulême, éditions de l'An 2.

Perronet, A. (2012). «L'étude d'un album de BD au cycle 3 : l'exemple de Petit vampire va à l'école ", in Rouvière, N. (dir), Bande dessinée et enseignement des humanités, Grenoble, ELLUG, p. 123-140. 
Rouvière, N. (2006). Astérix ou les lumières de la civilisation, Paris, PUF, 2006.

Rouvière, N. (2008). Astérix ou la parodie des identités, Paris, Flammarion.

Rouvière, N. (2008). «L'influence de la bande dessinée sur les albums pour enfants : histoire, esthétique et thématiques ", Modernités $n^{\circ} 28$, L'album contemporain pour la jeunesse : nouvelles formes, nouveaux lecteurs?, Presses universitaires de Bordeaux, p. 17-28.

Rouvière, N. (2012). Bande dessinée et enseignement des humanités, Grenoble, ELLUG.

Rouvière, N. (2012). «Etudier une œuvre intégrale en bande dessinée au cycle 3 : quelles spécificités didactiques ? ", in Bande dessinée et enseignement des humanités, Grenoble : ELLUG, p. 103-121

Rouvière, N. (2012) « Produire des planches pour apprendre à lire la BD : quelques dispositifs et leurs effets dans des classes de cycle 3 ", revue R2-LMM, vol. 1, 2015 ; article en ligne : http:// www.litmedmod.ca/produire-des-planches-pour-apprendre-lire-la-bd-quelques-dispositifs-etleurs-effets-dans-des

Rouvière, N. (2013). « Apprendre une lecture distanciée », in Les cahiers pédagogiques, n 506, A l'école de la bande dessinée, juin 2013, p. 34-38.

Rouvière, N. (2014). Le Complexe d'Obélix, Paris, PUF, 2014.

Rouvière, N. (2017). « Adapter en classe des extraits de roman sous forme de BD : quels apports pour la lecture littéraire? », in revue R2-LMM, vol. 6, Relations intersémiotiques en didactique des arts et de la littérature, https://litmedmod.ca/sites/default/files/pdf/r2-

lmm_vol5-6_rouviere.pdf

\section{NOTES}

1. Les références de ces ouvrages, comme celles des articles évoqués dans l'entretien, font l'objet d'une bibliographie à la suite de l'entretien.

\section{AUTEURS}

\section{NICOLAS ROUVIÈRE}

Université de Grenoble

\section{HÉLÈNE RAUX}

Université de Montpellier, Lirdef 